\title{
DEPLOYING MULTI-TIER APPLICATIONS ACROSS MULTIPLE SECURITY DOMAINS
}

\author{
Igor Balabine, Arne Koschel \\ IONA Technologies, PLC \\ 2350 Mission College Blvd \#1200 \\ Santa Clara, CA 95054 USA \\ \{igor.balabine, arne.koschel\} @iona.com
}

\begin{abstract}
In this paper we present an infrastructure layer, called Intermediary Security Platform (iSP), which provides multi-tier applications with a uniform abstraction of the authentication and authorization services. The abstraction is achieved via an intermediary Security Service (iS2). iS2 presents applications with a uniform interface for authentication and authorization requests. In turn, iS2 interfaces with Enterprise Security Systems (ESS) deployed at the site. iSP provides multitier application components authentication services, authorization services, and a single sign-on facility, all of which can bridge multiple security domains established at the site. At the same time, user management tasks are still performed by dedicated ESS. iSP architecture simplifies deployment of multi-tier applications on highly partitioned networks.
\end{abstract}

Keywords: authentication and authorization services, security architecture, multi-tier applications

\section{INTRODUCTION}

When an Enterprise application performs its tasks, it decides whether a request can be carried out based on the access rights assigned to the request originator. Information about access rights associated with the requestor is typically stored and maintained by the Enterprise Security System (ESS) [1].

For highly developed Enterprise infrastructures, it is typical to have more than one ESS, each of which maintains one or more security domains in which service requestor's access rights are defined [2]. For example, a financial services company may maintain a Web access security domain, which specifies access rights for Internet-based users, and another security domain for granting access to the backend systems.

The original version of this chapter was revised: The copyright line was incorrect. This has been corrected. The Erratum to this chapter is available at DOI: 10.1007/978-0-387-35696-9_19 
Proliferation of Web services requires the bridging of multiple Enterprise security domains, so that Internet-based users are presented with a robust and seamless view of the service provided by the Enterprise. Crossing security domain boundaries requires proliferation of the requestor's identity from one security domain to another, in a highly controlled and secure manner governed by individual domains' policies and rules. In many cases, a requestor's identity defined in one domain must be transformed for performing service in another domain, and access control is enforced by ESS with different capabilities [3].

\section{BRIDGING SECURITY DOMAINS VIA A SECURITY PLATFORM}

Security Platform (iSP) provides an abstraction layer that insulates applications from the authentication and authorization ESS, thus presenting a monolithic view of the security infrastructure. At the same time, iSP allows applications to use the existing Enterprise security infrastructure more easily, and makes possible orderly and secure transitions between different security domains. These transitions, which may involve impersonated and delegated actions, are achieved via a focal iSP component called Security Service (iS2). The following example of a Web services based multi-tier application, presented in Fig. 1 demonstrates iS2 functionality and capabilities.

In this scenario, a web-based customer communicates with a Web Services Gateway. Based on the content of the customer's message, the Gateway dispatches the request to a second-tier service, which is either a Web service or a J2EE application deployed on a J2EE-compliant application server. A second such service invokes a CORBA based middleware application, which in turn passes the request to a mission-critical Enterprise application deployed on a mainframe computer.

In this deployment, Enterprise security is enforced in two domains: the Front-End domain, in which Web applications are deployed, and the BackEnd domain, which hosts the Enterprise applications. The Front-end domain is served by a portal authentication and authorization system such as Netegrity SiteMinder, Evidian PortalXpert, etc. Windows Active Directory or Domain Controller, OS/390 RACF/ACF2/Top Secret access control facility, or a similar system governs security in the Back-End domain.

When a web-based customer establishes initial connection with the Web Services Gateway, she submits her credentials, e.g., user id and password or a digital certificate, which is valid in the Front-End security domain and managed by the portal ESS (1). The Gateway authenticates the customer with the Front-End authentication server and establishes customer's identity and authorizations in the Front-End security domain. As a consequence of this operation, the Gateway may receive a session authentication token, which it will return to 


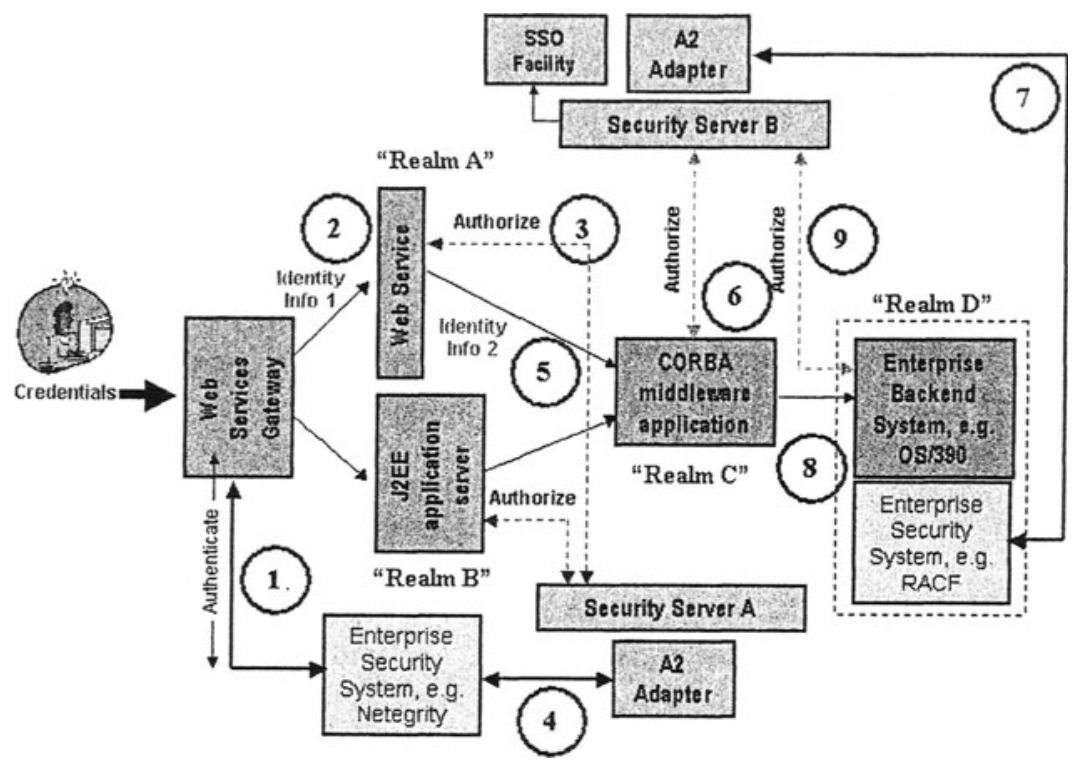

Figure 1 Multi-tier service spanning multiple security domains

the customer for use in subsequent invocations of the service. If the original user credentials or a portal authentication token is used to identify the Customer, then the steps described below are performed independently.

Once Customer's identity is established, the Web Services Gateway dispatches the request to one of the second-tier services. For simplicity we assume that the request is dispatched to the Web Service. The Web Services Gateway uses an application-level protocol to pass verified requestor's identity information to the Web Service (2). This application-level protocol is a private contract between the Web Services Gateway and the Web service, and allows passing the requestor's identity (that is, the principal) in a secure fashion, based on the trust relationship initially established by the parties. The contract is based on a public key or a shared secret method, and could have a proprietary or a normalized format. For example, this contract could be expressed as a SAML (Security Assertion Markup Language) [4] security assertion.

Upon receiving information about the requestor's identity (principal), the Web service issues an authorization request to the iS2 instance A (deployed in the Front-End security domain) asking for a list of access rights associated with 
this principal in its authority sub-domain, or realm, - called "Realm A" (3). iS2 uses a dedicated adapter to communicate with the portal authentication server and receives back a list of requestor's identities (principals) along with a list of user authorizations for the requested realm (4).

After processing the request, the Web service forwards it to the middleware CORBA application for more processing on the backend. At this point the request is crossing the security domain boundary, and the Web service selects an identity known in the Back-End security domain - which could be same as the requestor's identity (principal) in the Front-End domain, or another identity selected according to the Enterprise security policy (5).

In turn, the CORBA middleware application issues an authorization request to iS2 instance B, deployed on the Back-End security domain, asking it to provide requestor's authorizations (6). In this example, the ESS in the BackEnd security domain does not support the single sign on functionality. In order to provide the single sign-on function, the iS2 Single Sign On (SSO) facility is deployed with iS 2 instance B. In the above example OS/390 Remote Access Control Facility (RACF) provides authorization information (7) in the BackEnd security domain, and the SSO feature is supported by the iS2 SSO facility.

Upon verifying the authorizations, the CORBA middleware application dispatches the request to the mainframe Enterprise application, supplying an SSO authorization token in the CSIv2-compliant (Common Secure Interoperability) [2] security context of the IIOP message that contains the request (8). The IIOP interceptor verifies the SSO authentication token and receives authorization information from the iS2 instance B (9). The verified requestor's identity is propagated to the mainframe application, which engages standard RACF methods to authorize actions at the business decision level.

\section{OTHER ISP ADVANTAGES}

The above example shows how iS2-enabled applications can demonstrate seamless performance in a complex partitioned environment that provides endto-end security for a multi-tier service. Besides the features demonstrated in this example, use of an intermediate security platform infrastructure provides the following advantages:

- The applications can be easily moved between different security domains. Rather than having to make changes in the application's code, it can simply be reconfigured to point to a different iS2 instance. For example, one or both second-tier services could be moved to the Back-End security domain by simply reconfiguring the iS2 URL to point to the iS2 instance B. 
- iS2 enabled applications are completely neutral with respect to changes in the ESS provider. Configuring iS2 to use a different ESS adapter and configuring the ESS adapter itself accomplishes the change.

- iS2 ESS adapters are simple but very powerful. Custom iS2 ESS adapters allow combining multiple ESS providers and performing requestor's access rights aggregation across multiple security domains.

- All user management operations are performed via ESS tools commonly used by the IT personnel. iS2 and iS2 ESS adapters are easy to install and configure.

- iS2 allows to achieve uniformity of features provided by different ESS. The iS2 SSO facility provides out of the box single sign on functionality, and iS2 Authorization Manager allows implementation of Role Based Access Control (RBAC) in environments that lack authorization functionality [3].

- Applications' authentication and authorization queries are expressed as SAML (Security Assertions Markup Language) assertions [4]. The iS2 SDK binds the assertions to the transport protocols and secure communications between applications and iS2, according to the SAML security profiles guidelines [5]. SAML security profiles ensure that sensitive information contained in the authentication and authorization requests is protected from tampering and eavesdropping.

- Centralized communications between iS2 ESS adapters and ESS simplify multi-tier application deployment in firewall-partitioned networks. iS2 could be co-located on the network with ESS eliminating need for opening the inter-departmental firewall for proprietary transports often used by ESS (e.g. SiteMinder authentication protocol). iS2 enabled applications may use traditionally accepted http over the SSL and IIOP over the SSL transports to communicate with iS2.

\section{CONCLUSION}

Introduction of an intermediate security platform concept provides a flexible and robust infrastructure for abstracting authentication and authorization services for the Enterprise grade multi-tier applications. This intermediate security layer allows integration with virtually all Enterprise Security Services and may provide missing components in the environments devoid of the authorization and the single sign-on functionality.

IONA Technologies implemented the intermediate security platform concept as a framework built into its products. IONA Security Framework is currently being deployed at multiple customer sites. It provides a shareable single 
sign-on facility, PKI, and Enterprise Management Systems integration services to Enterprise applications.

\section{References}

[1] J. Adams. IBM Grid Computing Strategy. $2^{\text {nd }}$ ApGrid Workshop, Taipei, May 2002.

[2] The Globus Project. OGSA Security Roadmap, July 2002.

[3] R.S. Sandhu, and E.J. Coyne. Role-Based Access Control Models, IEEE Computer, Vol. 29, No. 2, Feb. 1996.

[4] Assertions And Protocol For The OASIS Security Assertions Markup Language (SAML). Committee Specification 01, 2002.

[5] CORBA Common Secure Interoperability version 2. Object Management Group, 2000.

[6] Bindings And Profiles For The OASIS Security Assertions Markup Language (SAML). Committee Specification 01, 2002. 